\title{
The vaporization behavior of carbon and hydrogen from the early global magma ocean
}

\author{
NATALIA SOLOMATOVA ${ }^{1}$ AND RAZVAN CARACAS ${ }^{2}$
}

${ }^{1}$ CNRS UMR 5276, ENS de Lyon Site Monod

${ }^{2} \mathrm{CNRS}$

Presenting Author: nsolomat@gmail.com

Estimating the fluxes and speciation of volatiles during the existence of a global magma ocean is fundamental for understanding the cooling history of the early Earth and for quantifying the volatile budget of the present day. Using firstprinciples molecular dynamics, we predict the vaporization rate of carbon and hydrogen at the interface between the magma ocean and the hot dense atmosphere, just after the Moon-forming impact. The concentration of carbon and the oxidation state of the melts affect the speciation of the vaporized carbon molecules (e.g., the ratio of carbon dioxide to carbon monoxide), but do not appear to affect the overall volatility of carbon. We find that carbon is rapidly devolatilized even under pressure, while hydrogen remains mostly dissolved in the melt during the devolatilization process of carbon. Thus, in the early stages of the global magma ocean, significantly more carbon than hydrogen would have been released into the atmosphere, and it is only after the atmospheric pressure decreased, that much of the hydrogen devolatilized from the melt. At temperatures of $5000 \mathrm{~K}$ (and above), we predict that bubbles in the magma ocean contained a significant fraction of silicate vapor, increasing with decreasing depths with the growth of the bubbles, affecting the transport and rheological properties of the magma ocean. As the temperature cooled, the silicate species condensed back into the magma ocean, leaving highly volatile atmophile species, such as $\mathrm{CO}_{2}$ and $\mathrm{H}_{2} \mathrm{O}$, as the dominant species in the atmosphere. Due to the greenhouse nature of $\mathrm{CO}_{2}$, its concentration in the atmosphere would have had a considerable effect on the cooling rate of the early Earth. 